\title{
Acupuncture as a Complementary Therapy for Cancer Care: Acceptability and Preferences of Patients and Informal Caregivers
}

\author{
Laura Tack ${ }^{1,2}$, Tessa Lefebvre ${ }^{1,2}$, Virginie Blieck ${ }^{3}$, Lieselot Cool ${ }^{1}$, Hans Pottel ${ }^{5}$, Koen Van Eygen ${ }^{4}$, \\ Sofie Derijcke ${ }^{6}$, Philippe Vergauwe ${ }^{7}$, Patricia Schofield ${ }^{8}$, Rebecca Chandler ${ }^{9}$, Pauline Lane ${ }^{9}$, \\ Tom Boterberg'², Philip R. Debruyne ${ }^{1,10, *}$
}

${ }^{1}$ Department of Medical Oncology, Kortrijk Cancer Centre, General Hospital Groeninge, Kortrijk, Belgium

${ }^{2}$ Department of Radiation Oncology, Ghent University Hospital, Ghent, Belgium

${ }^{3}$ College for Traditional Chinese Medicine \& Karel de Grote University College, Antwerp, Belgium

${ }^{4}$ Department of Haematology, Cancer Centre, General Hospital Groeninge, Kortrijk, Belgium

${ }^{5}$ Department of Public Health and Primary Care at Kulak, Catholic University Leuven Kulak, Kortrijk, Belgium

${ }^{6}$ Department of Pneumology, General Hospital Groeninge, Kortrijk, Belgium

${ }^{7}$ Department of Gastro-Enterology, General Hospital Groeninge, Kortrijk, Belgium

${ }^{8}$ Sheffield Hallam University, Sheffield, United Kingdom

${ }^{9}$ Faculty of Health, Education, Medicine and Social Care, Anglia Ruskin University, Chelmsford, United Kingdom

${ }^{10}$ School of Life Sciences, Anglia Ruskin University, Cambridge, United Kingdom

Received September 1, 2020

Revised February 11, 2021

Accepted February 21, 2021

Correspondence to

Philip R. Debruyne

Kortrijk Cancer Centre, General

Hospital Groeninge, Kortrijk, Belgium

E-mail Philip.Debruyne@azgroeninge.be
Background: Acupuncture can effectively manage cancer-related side effects, for both patients undergoing treatment and for cancer survivors. It may also be effective in managing physiological and psychological symptoms common among informal caregivers of cancer patients.

Objectives: The aim of this survey was to explore the acceptability and preferences of cancer patients, cancer survivors, and their informal caregivers in relation to acupuncture. Methods: The survey was conducted from $20^{\text {th }}$ November to $27^{\text {th }}$ November 2018. The questionnaire was developed to explore acceptability and preferences, including motivation, symptoms to be addressed, and practical issues (location, cost, etc.), in relation to acupuncture.

Results: The survey response rate was $94.5 \%$ in cancer patients and cancer survivors and $100 \%$ in caregivers. Acceptability of acupuncture was $34.5 \%(n=40 / 116)$ and $48.0 \%$ ( $n=26 / 54)$ in cancer patients and caregivers, respectively. About 52.5\% $(n=21 / 40)$ of patients preferred to undergo acupuncture at the day center clinic, whereas caregivers had no specific preference. Patients and cancer survivors would use acupuncture for symptoms of fatigue $(60 \%)$, listlessness $(57.5 \%)$, and pain $(47.5 \%)$. Informal caregivers expressed an interest in using acupuncture for their pain, stress, and sleeping difficulties $48.0 \%(n=26 / 54)$.

Conclusion: Cancer patients, cancer survivors, and informal caregivers would accept acupuncture as a complementary therapy. This openness and preference to acupuncture provide the foundations for this complementary therapy to be incorporated into holistic and supportive cancer care, both for patients and those supporting them.

Keywords: Acupuncture, Cancer, Informal caregiver, Complementary therapy, Cancerrelated side effect

\section{INTRODUCTION}

Although cancer therapies become more advanced over time, relief of cancer-related symptoms remains essential.
The adverse effects of cancer and cancer treatments include nausea, hot flashes, fatigue, drowsiness, pain, anxiety, and sleeping difficulties. These effects can be long term for cancer patients undergoing treatment and cancer survivors, 
affecting their quality of life, mental wellbeing, functionality, independence, and overall health [1-4].

Complementary therapies such as acupuncture and mindbody techniques are becoming increasingly popular treatment options for managing cancer-related symptoms when conventional treatment does not bring satisfactory relief or causes adverse effects [5]. Acupuncture has been used for the management of cancer-related symptoms in both supportive and palliative care settings [6-11]. However, information on patient-reported outcomes and satisfaction with acupuncture services within the oncology setting is limited. The effects of cancer further extend to informal caregivers, who provide unpaid support to their loved ones throughout their treatment and recovery. Supporting a loved-one with cancer can have significant health and wellbeing implications, including psychological distress, anxiety, depression, sleeping difficulties, fatigue, poor immune functioning, pain, and decreased quality of life [12]. Therefore, there is a clear need for cancer care that inclusively addresses treatment related adverse effects on patients in addition to addressing the needs of informal caregivers who support them.

Acupuncture provides a possible complementary therapy with the potential to relieve the adverse effects of treatment on both the patient [7,13-15] and informal caregivers [16]. Acupuncture involves penetrating the skin with thin stainless steel needles on specific locations of the body $[2,13]$. For patients being treated for cancer and for cancer survivors, International Guidelines (e.g. The National Comprehensive Cancer Network, NCCN; National Center for Complementary and Integrative Health, NCCIH; ADD e.g. UK Guidelines; ADD e.g. WHO Guidelines) advocate the use of acupuncture in conjunction with pharmacologic interventions as a "mind and body complementary health approach" to assist in the management of pain and fatigue [17-19]. As such, acupuncture is the most frequently used complementary therapy used in integrative oncology settings in Western countries [3].

While the mechanism of action for acupuncture is not yet fully understood, it is recognized as a relatively safe and effective intervention. Only a few non-serious adverse events such as hematoma/bleeding, needling pain, and orthostatic symptoms have been reported [2]. Given that acupuncture does not interfere with primary treatments [3], it can be safely integrated within current cancer treatment regimens [1]. Acupuncture can be an effective complementary therapy for managing cancer or cancer therapy-related symptoms and improving post-operative pain, chemotherapy-related nausea, vomiting, pain, depression, anxiety, drowsiness, fatigue, numbness or tingling, sleep disturbance, and hot flashes $[7,14,15]$. It could also offer relief from physiological and psychological symptoms in informal caregivers of cancer patients. Acupuncture has been found to be effective in relieving anxiety, several types of pain-related disorders such as chronic headache and migraine, gastroenterological problems, and cardiovascular conditions $[16,20,21]$.

Despite the evidenced benefits and recommendations from international guidelines, acupuncture is not appropriately integrated within the conventional cancer treatment regimen. This has been attributed to skepticism about the effectiveness of this complimentary therapy [22-24], more so among the elderly population, even though several trials found acupuncture to have some efficacy in elderly patients [25-27].

In addition to randomized controlled trials to establish the effectiveness of acupuncture, patient-reported outcomes offer valuable information. The novelty of this survey study lies within its prospective design and the inclusion of informal caregivers of cancer patients and cancer survivors. This study explores the preferences of cancer patients undergoing treatment, cancer survivors, and their informal caregivers to receive acupuncture and their acceptability toward the use of acupuncture as a complimentary therapy for the management of physical and psychological symptoms.

\section{MATERIALS AND METHODS}

\section{Study population and design}

Participants were recruited by a healthcare worker (Laura Tack, Tessa Lefebvre) at the day center clinic of the Organisation of European Cancer Institute (OECI)designated clinical cancer center of the General Hospital Groeninge in Kortrijk (Belgium). Patients with a histologically confirmed solid tumor or hematological malignancy (any stage and any type of treatment), those receiving primary treatment or receiving treatment after relapse, and cancer survivors were asked to participate. Caregivers of both groups were also included. All participants were over 18 years of age and spoke Dutch.

Participation in this study (94.5\%) was high, likely because of the process of data collection used. A previous study by our research group implementing a survey found participation rates within this context could be low [28]. Therefore, a healthcare worker completed surveys with respondents.

A questionnaire of six questions, based on relevant literature, was developed for this study. The questionnaire collected data on patient and caregiver demographics and treatment status and explored preferences and acceptability regarding acupuncture. It further explored symptom management. Patients and caregivers were also asked to identify symptoms for which they would opt for acupuncture management. Multiple choice responses and free text fields were included.

On completion of the questionnaire, those interested were provided with an information booklet on acupuncture and 
were offered an introductory acupuncture treatment by Virginie Blieck.

The questionnaire did not collect any personal identifiers and was completed anonymously. Written consent was not required as the participants were informed that by completion of the questionnaire their permission for their data to be used for the study is implied. The study findings were stored in a computer database in accordance with the Belgian data protection laws. This study was registered at General Hospital Groeninge (AZGS2018093) and was approved by the local ethics committee.

\section{Statistical analysis}

Descriptive statistical analyses were performed to present participant characteristics. The association between interest in acupuncture, gender, and age was explored statistically using a contingency table and a Pearson chi-square test. All analyses were conducted using Microsoft Office Excel 2013 (Microsoft, Inc., Redmond, WA) and IBM SPSS v.25 (SPSS, Inc., Chicago, IL) software.

\section{RESULTS}

\section{Study population characteristics}

A total of 172 questionnaire responses were obtained at a response rate of $94.5 \%(n=172 / 182)$. Two questionnaire responses were excluded from analysis as the participants did not meet the inclusion criteria. Of the remaining 170 responses, 62 were provided by patients receiving primary treatment; 41 by patients receiving treatment after relapse; 13 by cancer survivors; and 54 by informal caregivers.

Table 1. Patient demographic and clinical characteristics $(n=116)$

\begin{tabular}{|c|c|c|}
\hline & No. & $\%$ \\
\hline Age, y & 62.8 & \\
\hline Mean (range) & 21.0-88.0 & \\
\hline \multicolumn{3}{|l|}{ Sex } \\
\hline Men & 32 & 27.6 \\
\hline Women & 84 & 72.4 \\
\hline \multicolumn{3}{|l|}{ Patient type } \\
\hline Receiving primary treatment & 62 & 53.4 \\
\hline Receiving treatment after relapse & 41 & 35.3 \\
\hline Disease-free, out of treatment & 13 & 11.2 \\
\hline \multicolumn{3}{|l|}{ Malignancy } \\
\hline Breast & 43 & 37.1 \\
\hline Digestive & 20 & 17.2 \\
\hline Hematologic & 16 & 13.8 \\
\hline Urological & 15 & 12.9 \\
\hline Gynaecological & 11 & 9.5 \\
\hline Thoracic & 6 & 5.2 \\
\hline Other* & 10 & 8.6 \\
\hline \multicolumn{3}{|l|}{ Therapy } \\
\hline Chemotherapy & 114 & 98.3 \\
\hline Surgery & 80 & 69.0 \\
\hline Radiotherapy & 54 & 46.6 \\
\hline Immunotherapy & 27 & 23.3 \\
\hline Hormonal therapy & 11 & 9.5 \\
\hline
\end{tabular}

*Other: bone, brain, head and neck, unknown primary.

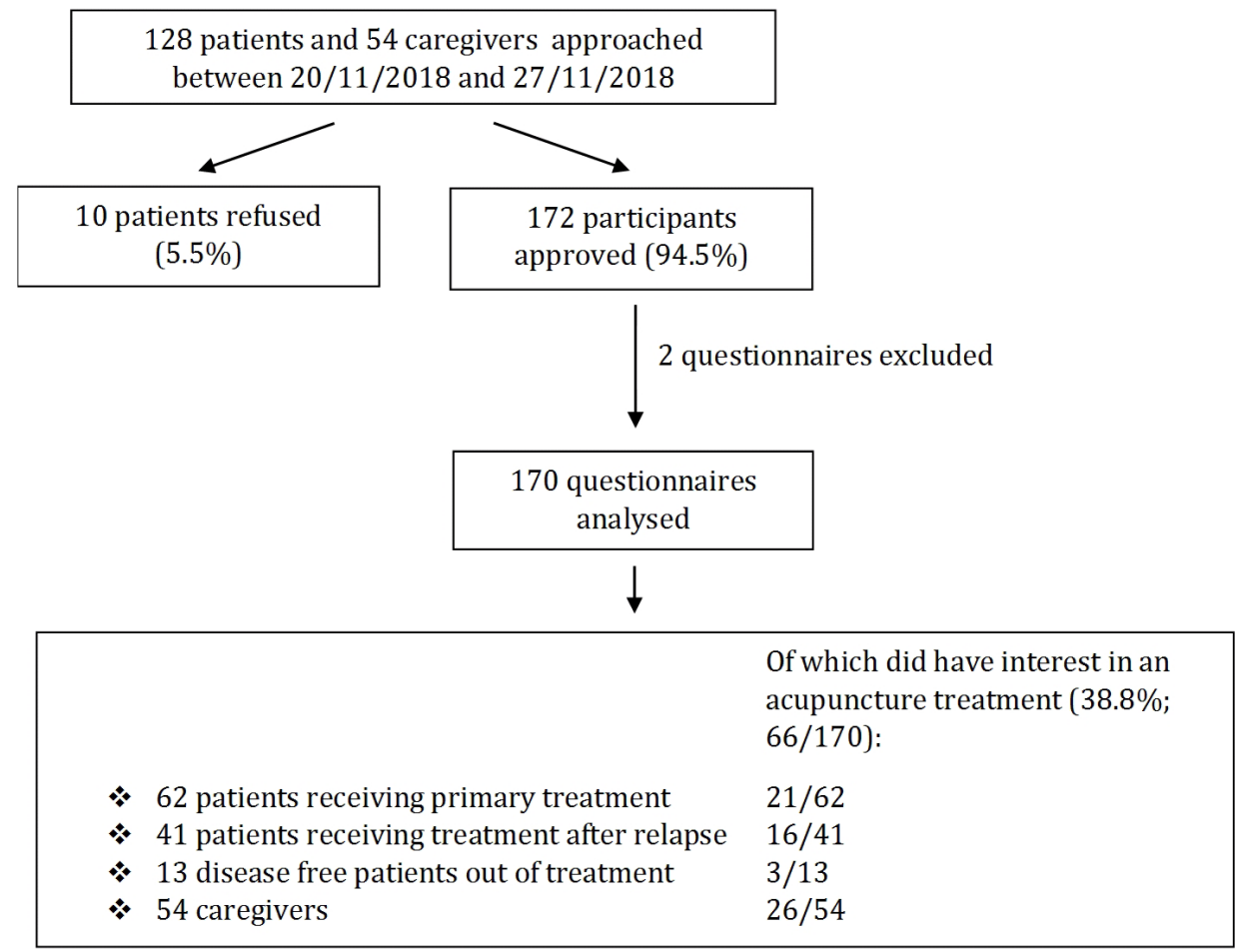

Fig. 1. Patient flowchart. 
Demographic and clinical characteristics of the patients are presented in Table 1 . The mean age of the patients was 62.8 years (range: $21-88$ years). Of the 116 patients, $72.4 \%$ were women, and $37.1 \%$ had been diagnosed with breast cancer. Almost all patients were treated with chemotherapy (98.3\%). Other therapies included surgery, radiotherapy, immunotherapy, and hormonal therapy $(69.0 \%, 46.6 \%$, $23.3 \%$, and $9.5 \%$, respectively). Some patients received multiple treatments.

\section{Acceptability and preferences of patients and cancer survivors}

In total, $38.8 \%$ of the participants were interested in acupuncture treatment $(n=66 / 170)$ (Fig. 1). Out of 116 patients and cancer survivors, $34.5 \%(\mathrm{n}=40 / 116)$ were interested in acupuncture treatment, whereas $65.5 \%$ were not ( $n=76 / 116)$. No association between gender and interest in acupuncture was found ( $p=0.67$ ) (Fig. 2A). However, the interest in acupuncture differed significantly between the youngest and oldest age categories $(p=0.004)$ (Fig. 2B). Those patients and cancer survivors aged 46 years or more, were least interested in acupuncture, whereas those aged 18-45 years were significantly more likely to accept acupuncture. Of all patient respondents, $16.4 \%(\mathrm{n}=19 / 116)$ were interested in receiving an introductory acupuncture treatment.

Of those patients interested in acupuncture treatment, $60.0 \%(n=40 / 66)$ indicated that they would be willing to selffund this complimentary therapy (Fig. 3A). Nevertheless, only $40.0 \%(n=16 / 40)$ of this group were interested in attending an information session about acupuncture (Fig. 3B).
A

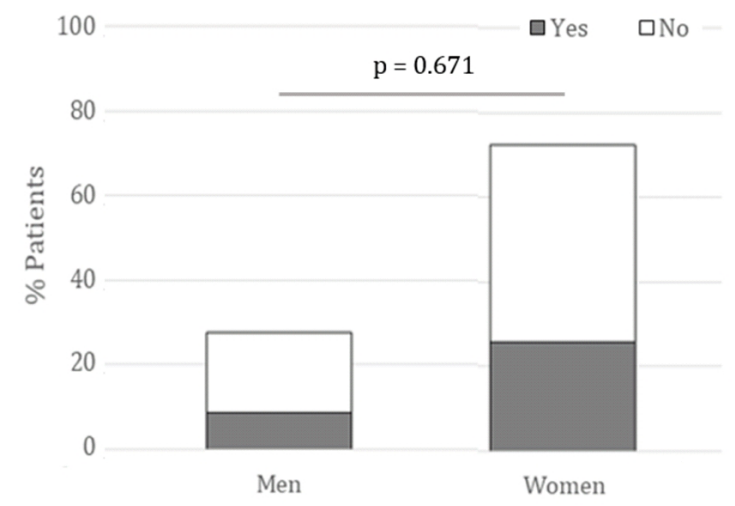

B

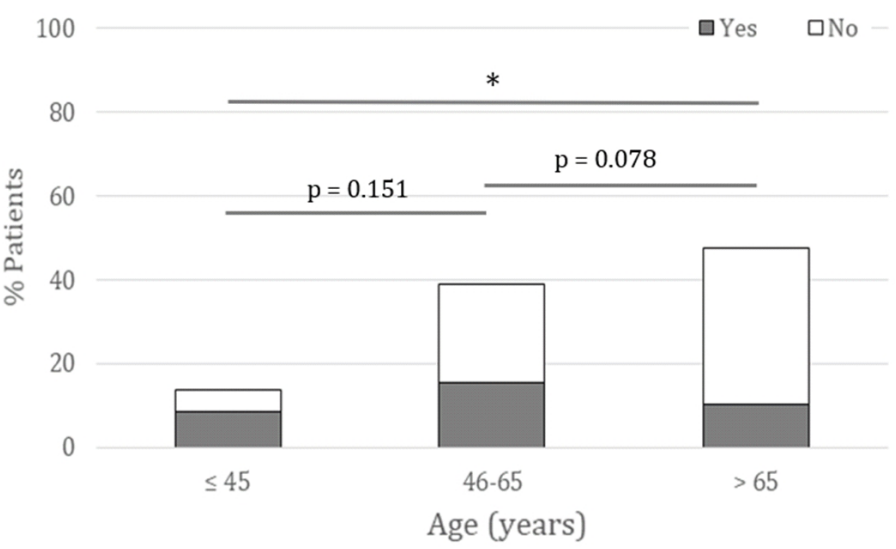

Fig. 2. (A) Patient interest in acupuncture stratified by gender. Total numbers of male and female patients were 32 and 84 , respectively. (B) Patient interest in acupuncture stratified by age. Total numbers of patients in the age groups of $\leq 45,46-65$, and $>65$ years were 16 , 45, and 55 , respectively. ${ }^{*} p<0.005$ by Pearson chi-square test.

A 100

80

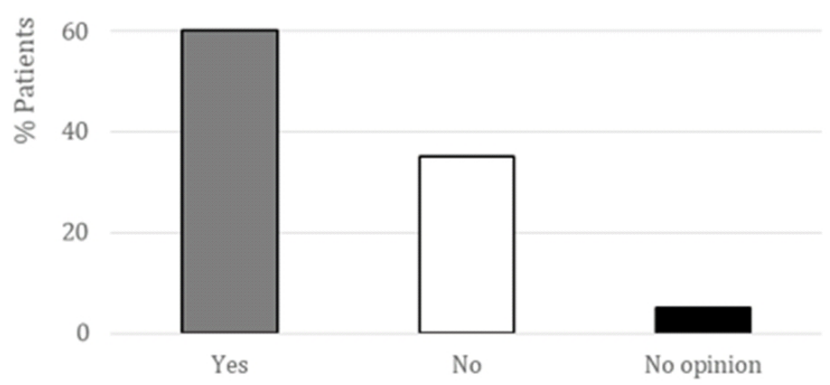

B

$$
100
$$

80

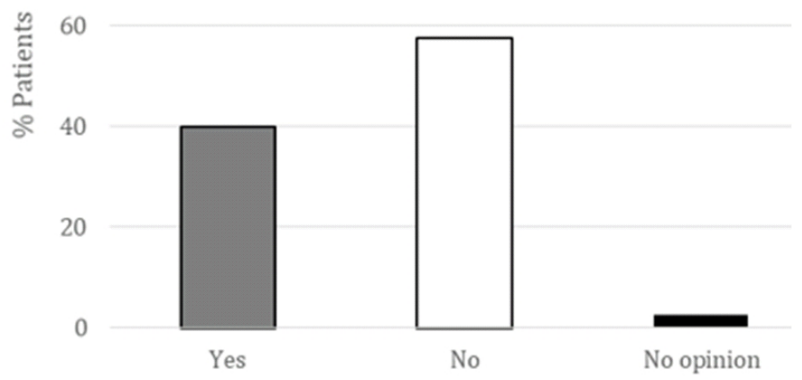

Fig. 3. (A) Proportions of patients who were willing to pay for acupuncture treatment. (B) Proportions of patients who were interested in an information session about acupuncture. 
100

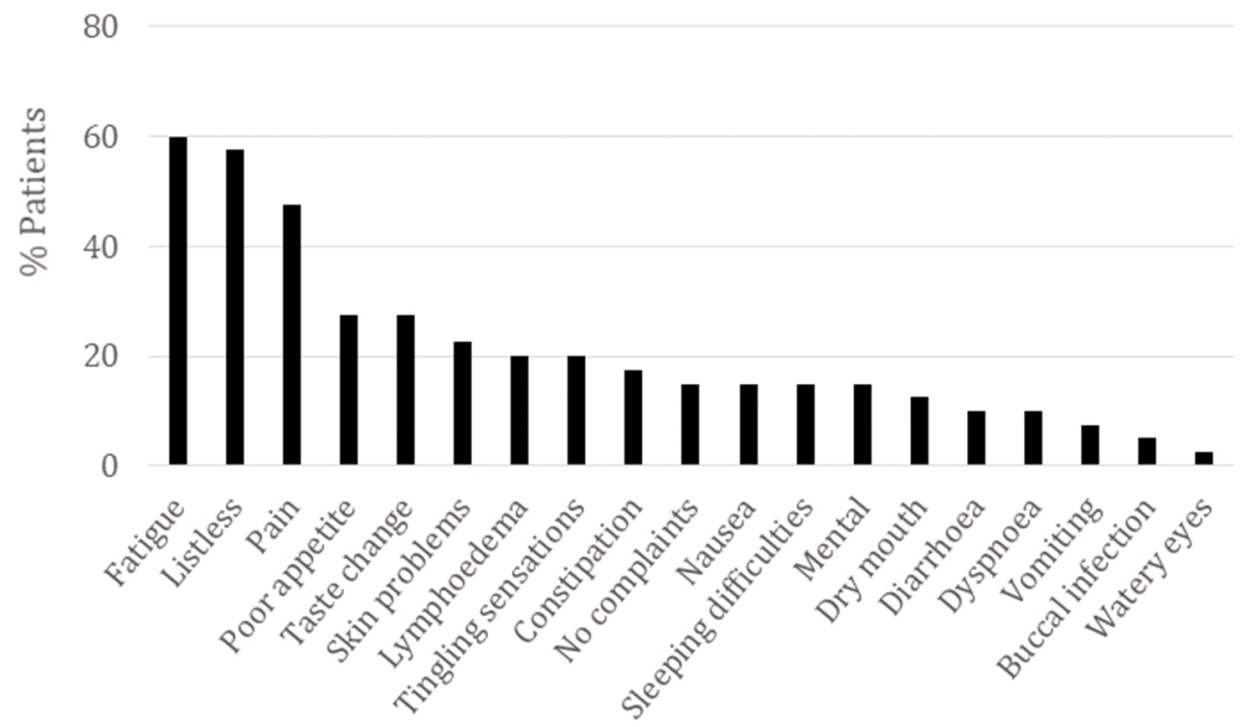

Fig. 4. Symptoms for which cancer patients and cancer survivors would receive acupuncture treatment.

100

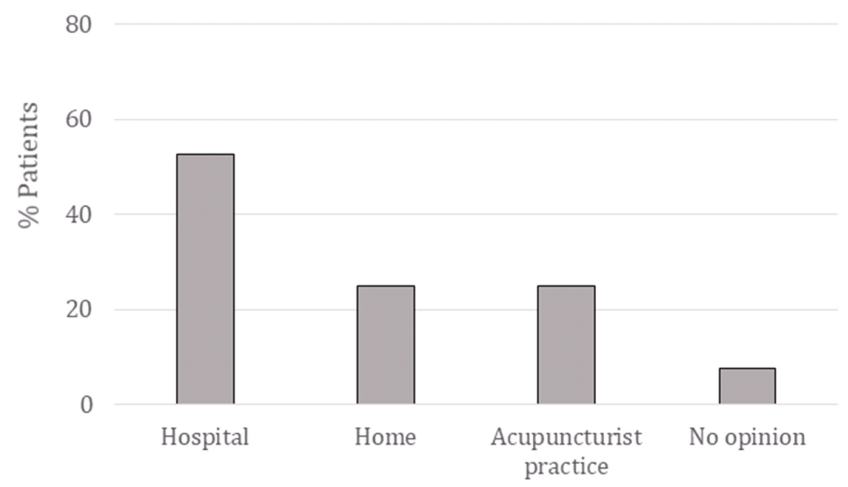

Fig. 5. Patients' preferences on locality of acupuncture treatment.

In terms of symptom management, patients and cancer survivors were interested in using acupuncture to address the following: fatigue $(60.0 \% ; \mathrm{n}=24 / 40)$, listlessness $(57.5 \%$; $\mathrm{n}=23 / 40)$, pain $(47.5 \% ; \mathrm{n}=19 / 40)$, poor appetite $(27.5 \% ; \mathrm{n}=$ $11 / 40)$, and taste change (27.5\%; $n=11 / 40)$ (Fig. 4). Other less frequently cited symptoms included oral and vocal problems; muscle weakness/stiffness/cramps; visual or sensory impairment; migraine; motor disturbance; nervousness; arthritis; bladder problems; heart issues; and smoking cessation.

To determine patient preferences regarding delivery of acupuncture treatment, the 40 interested patients and cancer survivors were asked about preferred locality of treatment (Fig. 5). A hospital was the most preferred locality $(52.5 \%$; $\mathrm{n}=$ $21 / 40)$, followed by home $(25.0 \% ; \mathrm{n}=10 / 40)$ and clinic of an accredited acupuncturist $(25.0 \% ; \mathrm{n}=10 / 40)$.
100

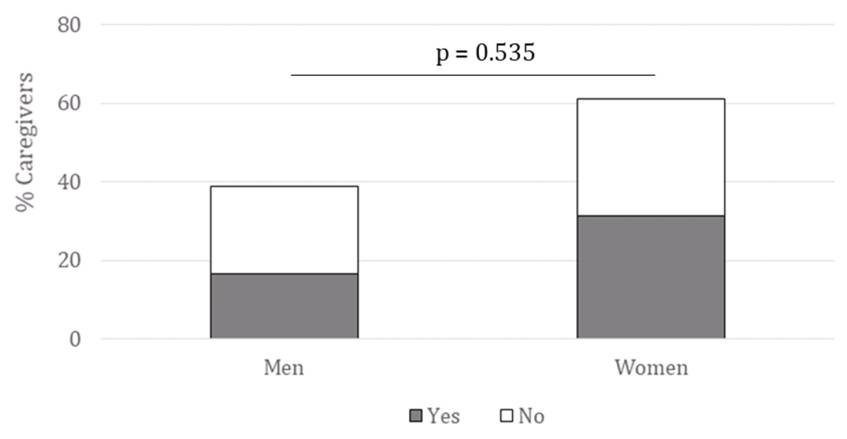

Fig. 6. Caregivers' interest in acupuncture stratified by gender.

\section{Acceptability and preferences of informal caregivers}

In total, $61.1 \%(\mathrm{n}=33 / 54)$ of informal caregivers were partners of patients, and $31.5 \%(\mathrm{n}=17 / 54)$ were relatives including siblings ( $41.2 \% ; n=7 / 17)$, parents $(17.6 \% ; n=3 / 17)$, and others $(41.2 \% ; n=7 / 17)$. The remaining were friends of patients and cancer survivors $(7.4 \% ; n=4 / 54)$. The mean age of all caregivers was 60.9 years (range: $28-83$ years).

Among caregivers, $48 \%$ were interested in acupuncture treatment $(n=26 / 54)$. There were more female respondents, but caregiver interest was not associated with gender $(p=0.54)$ (Fig. 6).

Symptom management in caregivers was not dissimilar to that in patients and cancer survivors. Pain was the most frequently mentioned symptom $(53.8 \% ; n=14 / 26)$, including headache, migraine, muscle pain, (low) back pain, nerve pain, shoulder pain, and arthritis. Other symptoms cited 
included: stress (23.1\%; $n=6 / 26)$, sleeping difficulties (19.2\%; $\mathrm{n}=5 / 26)$, lack of mental wellbeing (15.4\%; $\mathrm{n}=4 / 26)$, fatigue $(11.5 \% ; n=3 / 26)$, and stomach problems $(11.5 \% ; n=3 / 26)$. Many interested caregivers $(42.3 \% ; n=11 / 26)$ did not cite any specific symptoms they would wish to manage using acupuncture.

\section{DISCUSSION}

At present, integrative oncology programs combining complementary healthcare interventions such as acupuncture have become established in the United States and Europe $[29,30]$. With this survey study, we examined the acceptability to, and preferences for, acupuncture of patients undergoing treatment and cancer survivors as the information on patientreported outcomes and satisfaction with acupuncture services within the oncology setting is limited [31]. Moreover, we also included the opinions of 54 caregivers. Caregivers are often unjustly forgotten in the cancer treatment setting. Acupuncture is an alternative method for treating various diseases and symptoms.

This study found that $34.5 \%$ patients and cancer survivors would be interested in accepting acupuncture as an adjunctive and complementary therapy to their current cancer treatment or as standalone post-cancer treatment. Acceptability of acupuncture was associated with age, with those under the age of 45 years more likely to respond with interest than those over 46 years and especially over 65 years. This result is consistent with what we expected that older people would be more skeptic toward this treatment than younger people. There was no association between gender and acceptability. Interestingly, 57.5\% ( $n=23 / 40)$ of interested patients and cancer survivors did not wish to participate in an information session about acupuncture because they already had sufficient awareness of acupuncture and its benefits for symptom management.

Acceptability of acupuncture from patients and cancer survivors in this study is less than that observed in other studies. Lettner et al. found that $63.7 \%$ of German cancer patients receiving radiotherapy would use regular acupuncture treatment if it was integrated into the individual therapy concept [32]. This contrasting result to the current study may reflect the differences in countries, with Germany favoring natural products and complementary therapies compared to other European countries [32].

In terms of patient and cancer survivor preferences, over half were willing to self-fund (60\%) and preferred to receive an acupuncture treatment in a hospital setting (52.5\%). The respondents indicated that this was not only related to the safety of acupuncture but also because acupuncture was seen as an adjunct therapy that could be offered while waiting to receive other treatments.

Although 65.5\% ( $n=76 / 116)$ of patients were not interested in acupuncture treatment, $19.7 \%(\mathrm{n}=15 / 76)$ did wish to find out more by attending an information session.

The symptoms highlighted by respondents were diverse, encompassing cancer-related symptoms and wellbeing concerns, such as fatigue, pain, and oral complaints. Similar adverse effects were also reported by those with advanced cancer in a study by Kirkova et al. [33]. In their observational study in 181 patients with advanced cancer, fatigue was reported by $72 \%$ patients, followed by pain $(67.0 \%)$ and dry mouth (66.0\%) [33]. Indeed, the prevalence and severity of the symptoms highly depend on the primary site of disease and treatment modalities [34]. A subset of patients did not report any symptoms for which they would accept acupuncture treatment $(14.7 \% ; n=17 / 116)$, which could be because they had not yet initiated therapy.

Among caregivers, although more respondents expressed interest and willingness to accept acupuncture, over half did not (51.9\%). Moreover, no association between acceptability and gender was observed. Most caregivers (42.3\%; $n=11 / 26)$ did not report any symptoms for which they would use acupuncture. For those who did, pain, fatigue, stress, lack of mental wellbeing, and sleep disturbance were highlighted.

The novelty of this study lies within the exploration of the acceptability of cancer patients for acupuncture, whereas previous studies mostly focused on the use of acupuncture in pain management in cancer care or identified clinically meaningful differences. This survey study explored the opinions of patients undergoing cancer therapy and cancer survivors about acupuncture as a complementary therapy for managing cancer or cancer therapy-related symptoms. Moreover, the involvement of caregivers present at the day care clinic of the OECI-designated Kortrijk cancer center reflects the needs and acceptability for acupuncture of loved ones involved in cancer care. The results reflect how acupuncture could be implemented in an integrative oncology program.

This study also has some limitations. The survey was conducted only at one clinical cancer center in Belgium, and as such, we need to consider this limitation before applying our results on a wider scale. There is also a possibility of bias, which is inherent in all self-reported responses, in terms of participant reporting or responder bias to the healthcare worker conducting the survey.

\section{CONCLUSIONS}

This study provides insights into the preferences of cancer patients, cancer survivors, and their caregivers toward acupuncture treatment and their acceptability of this com- 
plementary therapy. Furthermore, the respondents mentioned diverse symptoms for which they would opt for acupuncture. Certainly, there is evidence of the efficacy of acupuncture in the treatment of psychological and physical symptoms. Acupuncture has the potential to be integrated with current cancer therapy regimens or administered to cancer survivors following cancer therapy to manage post-treatment adverse effects and disease symptoms. It may also be extended to caregivers who are integral to the recovery and wellbeing of patients (and indeed cancer survivors) but also have their own needs, which can be supported with acupuncture.

\section{ACKNOWLEDGEMENTS}

Our work was supported by the Belgian Foundation against Cancer (Stichting tegen Kanker, 2017-062).

\section{AUTHORSHIP STATEMENT}

All persons who meet authorship criteria are listed as authors, and all authors certify that they have participated sufficiently in the work to take public responsibility for the content, including participation in the concept, design, analysis, writing, or revision of the manuscript.

\section{AUTHORS' CONTRIBUTIONS}

Conceptualization: Tessa Lefebvre, Laura Tack, Philip R. Debruyne; Methodology: Tessa Lefebvre, Philip R. Debruyne; Validation: Tessa Lefebvre, Laura Tack, Hans Pottel, Philip R. Debruyne; Formal analysis: Tessa Lefebvre, Laura Tack, Philip R. Debruyne; Investigation: Tessa Lefebvre, Laura Tack, Virginie Blieck, Koen Van Eygen, Sofie Derijcke, Philippe Vergauwe, Philip R. Debruyne; Data curation: Tessa Lefebvre, Philip R. Debruyne; Writing - original draft preparation: Tessa Lefebvre, Laura Tack, Lieselot Cool, Philip R. Debruyne; Writing - review and editing: Tessa Lefebvre, Laura Tack, Virginie Blieck, Lieselot Cool, Hans Pottel, Koen Van Eygen, Sofie Derijcke, Philippe Vergauwe, Patricia Schofield, Rebecca Chandler, Pauline Lane, Tom Boterberg, Philip R. Debruyne; Visualization: Tessa Lefebvre, Laura Tack, Lieselot Cool, Philip R. Debruyne; Supervision: Patricia Schofield, Rebecca Chandler, Pauline Lane, Tom Boterberg, Philip R. Debruyne; Project administration: Tessa Lefebvre, Philip R. Debruyne; Funding acquisition: Tessa Lefebvre, Philip R. Debruyne.

\section{CONFLICT OF INTEREST}

The authors declare no conflict of interest.

\section{ORCID}

Laura Tack, https://orcid.org/0000-0002-0623-0160

Tessa Lefebvre, Virginie Blieck, Lieselot Cool, https://orcid.org/0000-0002-5267-5929 https://orcid.org/0000-0001-7622-6231 https://orcid.org/0000-0003-1435-3010 Hans Pottel, Koen Van Eygen, Sofie Derijcke, https://orcid.org/0000-0003-0074-8919 https://orcid.org/0000-0001-8285-906X https://orcid.org/0000-0003-4803-9193 Philippe Vergauwe, https://orcid.org/0000-0001-5046-6616 Patricia Schofield, https://orcid.org/0000-0003-4242-754X Rebecca Chandler, https://orcid.org/0000-0001-5479-537X Pauline Lane, https://orcid.org/0000-0003-4245-0504 Tom Boterberg, https://orcid.org/0000-0001-8680-8737 Philip R. Debruyne, https://orcid.org/0000-0001-5438-9697

\section{REFERENCES}

1. Lu W, Dean-Clower E, Doherty-Gilman A, Rosenthal DS. The value of acupuncture in cancer care. Hematol Oncol Clin North Am 2008;22:631-48, viii.

2. Lopez G, Garcia MK, Liu W, Spano M, Underwood S, Dibaj SS, et al. Outpatient acupuncture effects on patient self-reported symptoms in oncology care: a retrospective analysis. J Cancer 2018;9:3613-9.

3. Dobos GJ, Kirschbaum B, Choi KE. The Western model of integrative oncology: the contribution of Chinese medicine. Chin J Integr Med 2012;18:643-51.

4. Yabroff KR, Lawrence WF, Clauser S, Davis WW, Brown ML. Burden of illness in cancer survivors: findings from a population-based national sample. J Natl Cancer Inst 2004;96:132230 .

5. Deng G, Cassileth BR, Yeung KS. Complementary therapies for cancer-related symptoms. J Support Oncol 2004;2:419-26; discussion 427-9.

6. Javdan B, Cassileth B. Acupuncture research at Memorial Sloan Kettering Cancer Center. J Acupunct Meridian Stud 2015;8:11521.

7. Miller KR, Patel JN, Symanowski JT, Edelen CA, Walsh D. Acupuncture for cancer pain and symptom management in a palliative medicine clinic. Am J Hosp Palliat Care 2019;36:32632 .

8. Lyman GH, Greenlee H, Bohlke K, Bao T, DeMichele AM, Deng $\mathrm{GE}$, et al. Integrative therapies during and after breast cancer treatment: ASCO endorsement of the SIO clinical practice guideline. J Clin Oncol 2018;36:2647-55.

9. Chen H. Pancreatic cancer pain treatment with acupuncture. J Acupunct Meridian Stud 2020;13:69-70.

10. Zayas J, Ruddy KJ, Olson JE, Couch FJ, Bauer BA, Mallory MJ, et al. Real-world experiences with acupuncture among breast cancer survivors: a cross-sectional survey study. Support Care 
Cancer 2020;28:5833-8.

11. Ezzo JM, Richardson MA, Vickers A, Allen C, Dibble SL, Issell $\mathrm{BF}$, et al. Acupuncture-point stimulation for chemotherapyinduced nausea or vomiting. Cochrane Database Syst Rev 2006; (2):CD002285

12. Teixeira RJ, Applebaum AJ, Bhatia S, Brandão T. The impact of coping strategies of cancer caregivers on psychophysiological outcomes: an integrative review. Psychol Res Behav Manag 2018;11:207-15.

13. Garcia MK, McQuade J, Haddad R, Patel S, Lee R, Yang P, et al. Systematic review of acupuncture in cancer care: a synthesis of the evidence. J Clin Oncol 2013;31:952-60.

14. NIH Consensus Conference. Acupuncture. JAMA 1998;280: 1518-24.

15. Garcia MK, Cohen L, Spano M, Spelman A, Hashmi Y, Chaoul $\mathrm{A}$, et al. Inpatient acupuncture at a major cancer center. Integr Cancer Ther 2018;17:148-52.

16. Errington-Evans N. Randomised controlled trial on the use of acupuncture in adults with chronic, non-responding anxiety symptoms. Acupunct Med 2015;33:98-102.

17. National Comprehensive Cancer Network. Adult cancer pain 2019. Plymouth Meeting: National Comprehensive Cancer Network, 2019.

18. National Comprehensive Cancer Network. Cancer-related fatigue 2019. Plymouth Meeting: National Comprehensive Cancer Network, 2019.

19. National Center for Complementary and Integrative Health. Complementary, alternative, or integrative health: what's in a name? Available at: https://nccih.nih.gov/health/integrativehealth [Date accessed: June 3, 2019]

20. Pilkington K, Kirkwood G, Rampes H, Cummings M, Richardson J. Acupuncture for anxiety and anxiety disorders--a systematic literature review. Acupunct Med 2007;25:1-10.

21. Zhuang Y, Xing JJ, Li J, Zeng BY, Liang FR. History of acupuncture research. Int Rev Neurobiol 2013;111:1-23.

22. Harris LR, Roberts L. Treatments for irritable bowel syndrome: patients' attitudes and acceptability. BMC Complement Altern Med 2008;8:65.

23. Mallory MJ, Do A, Bublitz SE, Veleber SJ, Bauer BA, Bhagra A. Puncturing the myths of acupuncture. J Integr Med 2016;14:311-4.
24. Jackson S, Scambler G. Perceptions of evidence-based medicine: traditional acupuncturists in the UK and resistance to biomedical modes of evaluation. Sociol Health Illn 2007;29:412-29.

25. Mori H, Kuge H, Tanaka TH, Taniwaki E, Hanyu K, Morisawa T. Effects of acupuncture treatment on natural killer cell activity, pulse rate, and pain reduction for older adults: an uncontrolled, observational study. J Integr Med 2013;11:101-5.

26. Meng CF, Wang D, Ngeow J, Lao L, Peterson M, Paget S. Acupuncture for chronic low back pain in older patients: a randomized, controlled trial. Rheumatology (Oxford) 2003;42: 1508-17.

27. Qin Z, Ding Y, Xu C, Kwong JSW, Ji Y, Wu A, et al. Acupuncture vs noninsertive sham acupuncture in aging patients with degenerative lumbar spinal stenosis: a randomized controlled trial. Am J Med 2020;133:500-7.e20.

28. Lefebvre T, Tack L, Meersman M, Vanneste H, Cool L, Van Eygen K, et al. Developing and evaluating a participatory arts programme for cancer patients and their caregivers. Acta Clin Belg 2020. doi: 10.1080/17843286.2020.1773653. [Epub ahead of print]

29. Rossi E, Vita A, Baccetti S, Di Stefano M, Voller F, Zanobini A. Complementary and alternative medicine for cancer patients: results of the EPAAC survey on integrative oncology centres in Europe. Support Care Cancer 2015;23:1795-806.

30. Seely DM, Weeks LC, Young S. A systematic review of integrative oncology programs. Curr Oncol 2012;19:e436-61.

31. Thompson LM, Osian SR, Jacobsen PB, Johnstone PA. Patientreported outcomes of acupuncture for symptom control in cancer. J Acupunct Meridian Stud 2015;8:127-33.

32. Lettner S, Kessel KA, Combs SE. Complementary and alternative medicine in radiation oncology: survey of patients' attitudes. Strahlenther Onkol 2017;193:419-25.

33. Kirkova J, Walsh D, Rybicki L, Davis MP, Aktas A, Tao Jin, et al. Symptom severity and distress in advanced cancer. Palliat Med 2010;24:330-9.

34. Mazzotti E, Antonini Cappellini GC, Buconovo S, Morese R, Scoppola A, Sebastiani C, et al. Treatment-related side effects and quality of life in cancer patients. Support Care Cancer 2012; 20:2553-7. 